\title{
Frontières
}

\section{Les médias de masse sont-ils responsables des suicides en grappes?}

\section{Louise Pouliot}

Volume 21, numéro 1, automne 2008

Prévenir le suicide

URI : https://id.erudit.org/iderudit/037882ar

DOI : https://doi.org/10.7202/037882ar

Aller au sommaire du numéro

\section{Éditeur(s)}

Université du Québec à Montréal

ISSN

1180-3479 (imprimé)

1916-0976 (numérique)

Découvrir la revue

Citer cet article

Pouliot, L. (2008). Les médias de masse sont-ils responsables des suicides en grappes ? Frontières, 21(1), 120-125. https://doi.org/10.7202/037882ar
Résumé de l'article

Les médias de masse sont régulièrement mis en cause lorsque survient une série de suicides dans la population, dans les jours ou les semaines suivant le traitement médiatique du récit d'un cas de suicide. L'objectif de cet article n'est pas de démontrer ou de réfuter que les médias sont la cause de ces séries de suicide. Cet article présente une synthèse des résultats des recherches sur le lien entre les médias de masse et les suicides en grappes. L'article examine les hypothèses courantes avancées dans la littérature eu égard à ce lien et discute de la méthode empirique sur laquelle s’appuient les chercheurs pour étudier la relation entre les médias de masse et le suicide. Fondées sur la compréhension limitée que nous avons actuellement du phénomène, des pistes de solutions se situant à la fois au plan théorique et au plan méthodologique sont suggérées pour la recherche en ce domaine.
Ce document est protégé par la loi sur le droit d'auteur. L'utilisation des services d’Érudit (y compris la reproduction) est assujettie à sa politique d'utilisation que vous pouvez consulter en ligne.

https://apropos.erudit.org/fr/usagers/politique-dutilisation/ 


\section{Résumé}

Les médias de masse sont régulièrement mis en cause lorsque survient une série de suicides dans la population, dans les jours ou les semaines suivant le traitement médiatique du récit d'un cas de suicide. L'objectif de cet article n'est pas de démontrer ou de réfuter que les médias sont la cause de ces séries de suicide. Cet article présente une synthèse des résultats des recherches sur le lien entre les médias de masse et les suicides en grappes. L'article examine les hypothèses courantes avancées dans la littérature eu égard à ce lien et discute de la méthode empirique sur laquelle s'appuient les chercheurs pour étudier la relation entre les médias de masse et le suicide. Fondées sur la compréhension limitée que nous avons actuellement du phénomène, des pistes de solutions se situant à la fois au plan théorique et au plan méthodologique sont suggérées pour la recherche en ce domaine.

Mots clés: suicide - média - imitation vulnérabilité - psychologie des médias.

\begin{abstract}
Mass media are often blamed, in days and weeks following media treatment of a suicide story, for suicide epidemic outbreak. The goal of this article is not to refute or prove that media are the cause of suicide epidemics. It offers a synthesis of available research-based evidences on the link between mass media and suicide clusters, presents current forward hypotheses in the literature about the link, and discusses empiric method used by researchers to study such relation. Facing a limited understanding of the phenomenon, theoretical and methodological solutions are proposed.
\end{abstract}

Keywords: suicide - media - imitation vulnerability - media psychology.

\section{Les médias de masse sont-ils responsables des suicides en grappes?}

\author{
Louise Pouliot, Ph. D., \\ chercheure associée, CRISE, \\ Université du Québec à Montréal ${ }^{1}$.
}

L'histoire humaine a été marquée par deux révolutions technologiques importantes au chapitre de la diffusion de l'information. La première fut l'invention par Gutenberg, en 1437, de l'imprimerie et par John Baird, en 1926, de la télévision. Ces deux révolutions technologiques ont permis que les œuvres littéraires et cinématographiques, ainsi que les informations d'intérêt public rejoignent les masses. En parallèle, et ce très rapidement après leur invention et dérivant de leur usage comme canaux de communication, une croyance populaire s'est développée - et demeure toujours présente aujourd'hui - voulant que les messages qu'ils véhiculent sont la cause de la violence observée dans la population. La presse écrite et la télévision, en tant que médias de masse, sont également mises en cause dans les manifestations de violence autodirigée que sont les suicides, particulièrement lorsqu'ils surviennent en grappes dans les jours et semaines suivant le traitement médiatique du récit d'un cas de suicide.
À cet égard, et d'emblée, pour camper la discussion autour de la question principale qui est celle du lien entre les médias de masse et les séries de suicide, il est important de préciser que d'énoncer ou d'adhérer à l'idée que les médias de masse sont la cause des suicides en grappes serait une simplification. Plusieurs facteurs influencent le développement de la conduite suicidaire (Mishara et Tousignant, 2004). Les prédispositions biologiques peuvent augmenter ou diminuer la probabilité d'un geste suicidaire. Les caractéristiques de la famille, de la communauté, des pairs de l'individu peuvent stimuler, par le biais de la socialisation à poser de tels gestes. En outre, certains facteurs situationnels peuvent précipiter le suicide. Ainsi, l'objet principal de cet article n'est pas de démontrer que les médias sont responsables ou sont la cause des suicides en grappes, mais plutôt d'offrir une synthèse des résultats émanant des recherches sur le lien entre les médias de masse et ce phénomène. En parallèle, un corpus impressionnant de recherches dans le domaine de la psychologie des médias indique clairement que les productions médiatiques ont des effets sur les cognitions et les comportements des 
humains (Marchand, 2004; Giles, 2003; Singer et Singer, 2001). Ainsi, il est inutile de faire une telle démonstration. Cet article présente également les hypothèses les plus courantes avancées dans la littérature eu égard à ce lien. La méthode empirique à laquelle font appel la majorité des chercheurs pour étudier la relation entre les médias de masse et le suicide est aussi discutée. Enfin, la connaissance actuelle du phénomène est replacée dans le contexte de l'approche théorique et de la tradition de recherche qui marquent l'étude du lien entre les médias et les suicides en grappes.

\section{QUELQUES ÉLÉMENTS DE DÉFINITION}

L'examen des écrits scientifiques sur les suicides en grappes nous amène au constat qu'il n'y pas de définition qui fasse consensus. Néanmoins, la définition proposée par les Centers for Disease Control des ÉtatsUnis (O'Carroll et al., 1988) est celle qui est le plus souvent employée dans la littérature scientifique. Suivant cette définition: «Les suicides en grappes peuvent être définis comme un groupe de suicides ou de tentatives de suicide se produisant de manière proximale dans le temps et l'espace, et dont l'occurrence dans une communauté excède les probabilités » (traduction libre, p. 1). À la lecture de cette définition, on constate rapidement un flou important quant aux critères spécifiques d'inclusion de cas de suicides dans les grappes. Notamment, elle n'explicite pas les paramètres temporels et spatiaux dont il faut tenir compte pour cerner le phénomène des suicides en grappes, ce qui laisse aux chercheurs une latitude évidente pour fixer ces mêmes paramètres. De même, le seuil de probabilité n'est pas fixé dans la définition, ouvrant la voie à l'interprétation des chercheurs. Ces éléments ne sont pas étrangers à certains problèmes rencontrés dans les études qui ont porté sur le lien entre le traitement dans les médias du récit d'un cas de suicide et les suicides en grappes.

Les manifestations des suicides en grappes ne sont pas fondées sur un seul processus ou trajectoire. D'ailleurs, Joiner (1999) propose de faire la distinction entre deux types de suicides en grappes. Suivant la typologie de Joiner (1999), il y a d'abord les grappes de masse, associées à la présentation dans les médias (presse écrite, livres, télévision) du récit d'un cas de suicide et produisant généralement, selon certains auteurs, des séries de suicides dans les sept jours suivants (Phillips et Cartensen, 1986; Bollen et Phillips, 1982). De plus, ces suicides se concentrent habituellement dans la zone géographique qui est couverte par les médias qui ont diffusé le récit du cas de suicide (p. ex. : ville, province, État, pays). Le second type, dans la typologie de Joiner (1999), correspond aux grappes localisées, dont les suicides sont concentrés dans le temps, l'espace, ou au sein d'un groupe de personnes. Les exemples typiques de ce type de grappes sont les séries de suicides qui surviennent à l'intérieur d'une même école ou d'une même collectivité.

\section{CE QUE LES DONNÉES SCIENTIFIQUES DISENT AU SUJET DU LIEN ENTRE LES MÉDIAS DE MASSE ET LE SUICIDE}

Dans son traitement des grappes de masse, Phillips (1974) désigne l'effet des traitements médiatiques de récits de cas de suicide sur les comportements suicidaires dans la population par l'appellation de «l'effet Werther», en référence à l'augmentation dramatique des suicides par arme à feu en Europe qui semblent être survenue à la suite de la publication de l'œuvre classique de Goethe Les souffrances du jeune Werther (1774). Le roman de Goethe raconte l'histoire d'un jeune homme qui se tue à l'aide d'une arme à feu à la suite d'une peine d'amour.

Au cours des quarante dernières années, un nombre appréciable d'études rapportent que le traitement médiatique de récits de suicides est associé à une hausse du nombre de suicides dans la population, particulièrement lorsque le récit porte sur le suicide d'une personne célèbre (p. ex. : Romer et al., 2006; Tousignant et al., 2005; Etzerdorfer et al., 2004; Frei et al., 2003; Schmidtke et Schaller, 1998; Stack, 1996; Hassan, 1995; Jonas, 1992; Fekete et Macsai, 1990; Berman, 1988; Gould et al., 1988; Schmidtke et Häfner, 1988; Stack, 1987; Gould et Shaffer, 1986; Phillips et Cartensen, 1986; Bollen et Phillips, 1982; Phillips, 1974). Toutefois, un nombre plus restreint d'études ne rapportent pas une telle association, par exemple l'étude de Littmann (1985) qui s'appuie sur une méthode très différente et l'étude de Kessler et ses collègues (1988), qui s'avère une contre-vérification de la recherche de Phillips et Carstensen (1986), mais qui comporte des modifications au niveau de plusieurs des paramètres d'analyse. À cette liste de recherches s'ajoutent celles de Platt (1987), de Martin et Koo (1997) et de Phillips et Paight (1987) qui n'indiquent pas un tel «effet», et des recherches de Hittner (2005) et de Shoval et ses collègues (2005) qui n'offrent qu'un support partiel à «l'effet Werther».

En parallèle, le corpus de recherches sur «l'effet Werther» met en évidence des variations dans la magnitude de l'effet selon le type de médias à partir duquel l'histoire de suicide est traitée (médias écrits ou audiovisuels) et selon la nature fictive ou réelle de l'histoire de suicide traitée. L'association apparaît plus fréquemment dans le cadre des études portant sur les reportages qui présentent des cas de suicide réels dans la presse écrite, que lorsqu'il s'agit d'études portant sur un autre type de média ou portant sur des cas fictifs. De plus, «l'effet Werther» ne semble pas se limiter à un contexte culturel particulier, puisqu'il a été observé dans plusieurs cultures, dont en Hongrie (Fekete et Macsai, 1990), en Allemagne (Schmidtke et Häfner, 1988), au Québec (Tousignant et al., 2005), en Chine (Yip et al., 2006), en Australie (Hassan, 1995; Pirkis et al., 2006), au Japon (Stack, 1996) et aux États-Unis (Phillips et Carstensen, 1986). Cependant, cela ne signifie pas pour autant que la culture n'a pas d'impact sur le phénomène.

\section{COMMENT LES CHERCHEURS EXPLIQUENT-ILS LE LIEN ENTRE LES MÉDIAS DE MASSE ET LE SUICIDE ?}

Dans la littérature scientifique, on a recours principalement à deux explications pour comprendre le phénomène des suicides en grappes reliés aux médias de masse. En premier lieu, plusieurs chercheurs font appel à l'hypothèse de l'imitation. L'hypothèse est tirée de la théorie de l'apprentissage social d'Albert Bandura (1977). En résumé, cette théorie soutient que l'observation d'un comportement chez un modèle peut favoriser, sous certaines conditions, l'imitation (ou en d'autres termes la reproduction) du même comportement chez celui qui observe le modèle. De plus, suivant la théorie de Bandura, les conditions qui augmentent ou réduisent la probabilité que l'imitation du comportement du modèle par l'imitateur prenne place, sont l'identification ou non au modèle, les conséquences (positives ou négatives) associées au comportement du modèle et la fréquence ou le dosage avec lequel l'imitateur est exposé au comportement du modèle. Dans le contexte des grappes de masse, on soutient, à quelques variantes près de l'hypothèse de Bandura, qu'une personne qui est exposée au geste suicidaire d'un individu par l'intermédiaire des médias peut être susceptible de copier le même geste suicidaire. Or, les chercheurs ne considèrent que très rarement les corollaires importants de l'imitation, dont l'identification, les conséquences associées au comportement du modèle et le dosage de l'exposition au comportement du modèle. Notamment, conformément aux prémisses de la théorie de Bandura, les individus ont plus tendance à modeler leurs comportements sur celui de modèles 
auxquels ils peuvent s'identifier par leurs similitudes au plan sociodémographique (âge et genre), ou à cause du prestige qu'ils représentent. De plus, les personnes vont traduire en action le comportement des modèles lorsque ces comportements présentent ou mènent à des conséquences positives. Finalement, la reproduction par l'individu du comportement est plus probable lorsqu'il est exposé de façon répétée aux conduites d'un modèle ou de modèles différents dont les conséquences observées sont positives.

Les résultats de quatre études (Tousignant et al., 2005; Schmidtke et Häfner, 1988; Fekete et Macsai, 1990 ; Ashton et Donnan, 1979), bien qu'offrant des évidences indirectes touchant l'hypothèse de l'imitation, appuient sa validité. Ces études indiquent, pour certains cas de suicides observés subséquemment au traitement dans les médias du récit d'un cas de suicide, que les personnes emploient le même moyen pour mettre fin à leurs jours que celui utilisé par le protagoniste présenté dans le récit. La méta-analyse de Stack (2005) reprenant les 419 observations de 55 études quantitatives portant sur l'effet du traitement médiatique de cas réels de suicides ne supporte pas quant à elle l'hypothèse d'imitation. Selon la méta-analyse de Stack (2005), 64,5\% des observations infirment l'hypothèse. Mais la méta-analyse de Stack (2005) présente certaines limites méthodologiques susceptibles de nuancer la portée des résultats que rapporte l'auteur. L'auteur de la métaanalyse combine des études ayant porté sur des récits de cas de suicides traités à partir de différents médias (presse écrite et électronique) et dont les protagonistes n'ont pas le même profil sociodémographique (en termes d'âge, de genre, d'ethnie, de statut social, etc.). Par conséquent, les résultats de l'auteur auraient pu aller dans une tout autre direction en raison de l'hétérogénéité des stimuli à la base des études combinées dans la méta-analyse. Ainsi, en raison de la nature des preuves et du faible nombre d'études qui pointent en direction d'un processus de l'ordre de l'imitation, cette hypothèse ne s'avère pas encore validée.

Des auteurs suggèrent que certaines personnes sont plus prédisposées que d'autres à poser un geste suicidaire en conséquence du traitement dans les médias d'une histoire de suicide en raison de vulnérabilités préalables (p. ex. : Chiu et al., 2007 ; Zahl et al., 2004; Doron et al., 1998; Fekete et Schmidtke, 1996). En d'autres termes, ces personnes posséderaient une ou plusieurs caractéristiques personnelles (ou vulnérabilités) les rendant plus susceptibles à «l'effet Werther». Cet effet, plus probable chez les personnes vulnérables ou ayant des prédispositions, pourrait s'expliquer à partir du modèle de la sensibilisation (Post et Weiss, 1995 ; Post, 1992). Selon ce modèle, les personnes vulnérables ou prédisposées possèdent un seuil de tolérance au stress amoindri, occasionné notamment par l'exposition répétée à des événements psychosociaux et aux changements biologiques que cette exposition provoque. En appliquant ce modèle au phénomène de "l'effet Werther», chez les personnes vulnérables, et ce, en raison de leur plus grande réactivité acquise au stress, elles s'avéraient plus susceptibles d'être influencées par la stimulation psychosociale que représente l'exposition à une histoire de suicide dans les médias.

Les connaissances actuelles sur les caractéristiques (ou vulnérabilités) des personnes ayant complété un suicide suite à un effet des médias sont très limitées. Peu d'études ont examiné cette question et les résultats contradictoires ne permettent pas encore de valider cette hypothèse (Gould, 2001). Cependant, en ce qui concerne les caractéristiques individuelles des personnes s'étant suicidées dans le cadre d'une grappe de masse (Joiner, 1999), il ressort, et ce sur la base de trois études, que les personnes ayant fait une tentative de suicide dans le passé sont plus portées à poser un geste suicidaire suivant la présentation dans les médias du récit d'un cas de suicide (Cheng et al., 2007; Zahl et al., 2004; Fekete et Schmidtke, 1996; Martin, 1996).

\section{COMMENT LES CHERCHEURS ÉTUDIENT-ILS LE LIEN ENTRE LES MÉDIAS DE MASSE ET LE SUICIDE ?}

La majorité des preuves de l'existence des grappes de masse sont tirées d'études épidémiologiques (Stack, 2002, 2003 ; Phillips, Lesyna et Paight, 1992). La méthode consiste principalement à comparer le taux de suicide qui prévaut après le traitement dans les médias de l'histoire de suicide aux taux établis pour une ou plusieurs périodes antérieures à ce traitement. Cette méthode présente deux limites principales.

Tout d'abord, les preuves issues d'une telle approche méthodologique sont peu robustes, lorsque celle-ci est appliquée dans sa forme la plus simple. Plusieurs éléments du contexte social entourant la présentation des récits de suicides dans les médias ne sont pas contrôlés, tels que la qualité des services de soins de santé, les conditions socioéconomiques, les tendances saisonnières, les congés fériés, les changements dans la structure et la taille des populations et les taux de chômage
(Gould, 2001; Pirkis et Blood, 2001b ; Schmidtke et Schaller, 1998). De tels éléments du contexte social sont susceptibles d'expliquer à eux seuls les fluctuations dans les taux de suicide, en dehors de "l'effet» du traitement de récits de cas de suicides dans les médias.

Ensuite, les preuves ne sont pas toujours directes. La plupart des études infèrent que la série de suicides observée à la suite du traitement dans les médias du récit du cas de suicide est la conséquence de ce traitement médiatique, sans toutefois offrir la preuve que les personnes de cette série de suicides ont bel et bien été exposées à l'histoire (Hawton et Williams, 2001 ; Pirkis et Blood, 2001a, 2001b). L'étude de Tousignant et ses collègues (2005) se démarque de plusieurs autres études sur la question par le recueil de preuves plus directes concernant l'impact de la couverture médiatique du suicide d'un journaliste bien connu survenu en 1999 au Québec. S'appuyant sur l'analyse des rapports des coroners pour les cas observés dans la série de suicides, les chercheurs établissent que plusieurs des personnes ont eu recours à la même méthode singulière de suicide employée par le journaliste, soit celle de la pendaison à l'aide d'une ceinture. En outre, cette analyse met en lumière que pour un nombre important des cas de suicide de la série, la famille a fait mention que leur proche décédé avait été fortement bouleversé ou influencé par la nouvelle du suicide du journaliste en question.

Enfin, d'autres aspects des études produites sur la question portent ombrage à la validité de «l'effet Werther». Les paramètres spatiaux et temporaux, de même que les critères d'inclusion des comportements suicidaires pour définir l'unité d'étude (c.-à-d. la grappe de masse) diffèrent sensiblement d'une recherche à l'autre. Ces éléments sont à même d'expliquer les résultats contradictoires entre les études. Or, la variabilité entre les études en ce qui concerne les paramètres utilisés et les critères d'inclusion n'est sans doute pas étrangère à l'absence d'une définition standardisée du phénomène des suicides en grappes (Hazell, 1993; Gibbons et al., 1990; Gould et al., 1990).

Les études de Gould et Shaffer (1986), Phillips et Paight (1987), Tousignant et ses collègues (2005), et Cheng et ses collègues (2007) peuvent servir d'illustrations de ce problème dans la définition même du phénomène. Gould et Shaffer (1986) analysent les cas de suicides et de tentatives de suicides d'adolescents admis dans les hôpitaux de l'État de New York pendant les deux semaines précédant et suivant la diffusion de quatre films relatant des suicides. Phillips et Paight (1987), dans leur contre- 
LES ÉCRITS PORTANT SUR L'EFFET DE CONTAGION ATTRIBUABLE

AUX RÉCITS DE CAS DE SUICIDES DIFFUSÉS PAR LES MÉDIAS

SOUFFRENT D'UNE MÉCONNAISSANCE DES THÉORIES

ET DÉCOUVERTES PLUS CONTEMPORAINES SUR LA RÉCEPTION

ET LE TRAITEMENT DES COMMUNICATIONS MÉDIATISÉES.

vérification de l'étude de Gould et Shaffer (1986), emploient comme unité d'analyse temporelle les six semaines précédant et suivant la diffusion des mêmes films et, comme unité d'analyse spatiale, les États de la Californie et de la Pennsylvanie. La contre-vérification par Phillips et Paight (1987) de l'étude antérieure de Gould et Shaffer (1986) n'inclut que les suicides complétés. Plus récemment, Tousignant et ses collègues (2005) comparent les données populationnelles québécoises sur les suicides observés dans les 30 jours suivant le traitement par la presse écrite $\mathrm{du}$ suicide par pendaison du journaliste Gaétan Girouard en 1999, à celles observées durant la même période pour deux années témoins. Cheng et ses collègues (2007) comparent les tentatives de suicide observées dans les trois semaines suivant la couverture médiatique du suicide d'un acteur célèbre taïwanais, en 2005, à celles observées pour les 34 premières semaines de deux années témoins.

Par ailleurs, d'un point de vue méthodologique, les études qui ont porté sur le phénomène des grappes de masse n'offrent pas un support très solide de «l'effet Werther», car la relation de cause à effet n'est pas en soi établie, et ce, en raison de la nature indirecte des preuves. De plus, dans ces études, plusieurs facteurs d'ordre contextuel et statistique ne sont pas contrôlés (p. ex.: la dépendance statistique entre les suicides attendus et observés liée à une troisième variable comme l'économie; voir Hittner, 2005), lesquels facteurs peuvent expliquer les liens observés et rapportés.

$$
\star \star \star
$$

Après avoir passé en revue les preuves avancées dans les écrits sur la relation entre le traitement dans les médias de récits de cas de suicides et les suicides en grappes, nous en concluons que cette relation est plus systématiquement observée lorsqu'il s'agit de la presse écrite que des autres médias bien que, en raison de la nature des preuves, il n'est pas possible de dire que l'un ou l'autre des médias de masse a un plus grand «effet».

Nous en savons très peu au sujet des mécanismes psychologiques impliqués dans «l'effet» des traitements médiatisés de récits de cas de suicides sur les comportements suicidaires. Les hypothèses de la vulnérabilité préalable et de l'imitation ont reçu quelques appuis indirects. La méthode empirique de nature corrélationnelle employée par les chercheurs pour étudier la question comporte son lot de limites et, dans sa forme la plus simple, rend peu probable une progression dans notre compréhension du phénomène.

Les écrits portant sur l'effet de contagion attribuable aux récits de cas de suicides diffusés par les médias souffrent d'une méconnaissance des théories et découvertes plus contemporaines sur la réception et le traitement des communications médiatisées. Elle reste ancrée dans la tradition des "effets des médias» des années 1960 et 1970, alors qu'à l'époque, l'ensemble des efforts en recherche consistait à établir un lien entre certains contenus médiatiques et certains effets psychologiques et comportementaux. Depuis lors, la recherche dans le domaine de la psychologie des médias a établi que les auditoires ne sont pas des récepteurs passifs des médias. Ils jouent un rôle actif tant dans leur exposition aux médias que dans l'interprétation qu'ils donnent au contenu médiatique. Cette exposition est notamment conditionnée par leurs besoins et motivations: besoin de s'informer, de se divertir, d'affiliation, de briser leur isolement, etc. Leurs interprétations des contenus médiatiques sont intimement associées à leur connaissance préalable des caractéristiques des différents médias de communication, de leurs connaissances du monde social en général, mais aussi par leurs styles cognitifs ${ }^{2}$.

Le travail à effectuer pour contribuer à l'avancement des connaissances sur le phénomène de «l'effet Werther» se situe à deux niveaux. D'abord, au plan théorique, il importe de formuler un modèle qui allie à la fois les connaissances cumulées dans le domaine de la psychologie des médias de masse à celles de la suicidologie, et de voir à sa vérification.

Un tel modèle, d'une part, devrait intégrer des éléments de connaissance liés aux caractéristiques propres des différents médias de masse, au traitement cognitif de l'information médiatisée et aux traits de personnalité des récepteurs. La psychologie des médias de masse a démontré que ces deux derniers types d'éléments jouent un rôle modérateur dans l'effet des médias en général sur la conduite humaine.

D'autre part, un tel modèle devrait considérer l'état des connaissances en suicidologie relativement aux principaux facteurs de risques au suicide, touchant tant des dimensions biologiques, psychosociales, que culturelles des individus. $\mathrm{Au}$ surplus, ce modèle devrait s'appuyer sur des connaissances factuelles et des résultats probants fournis par des études fondées sur la méthode scientifique, en priorisant notamment celles qui ont été contre-vérifiées, issues d'étude ayant un échantillon de bonne taille et dérivant d'une démarche rigoureuse pour l'obtention des données (opérationnalisation des concepts étudiés, contrôle maximal des facteurs exogènes ou concomitants à l'objet d'étude, etc.).

Le second niveau est celui de l'approche méthodologique. Il importe d'adopter une approche de triangulation, combinant des méthodes de recherche et différents types de mesures. Sur le plan du comportement, et ce, pour des raisons éthiques évidentes en raison du phénomène sous étude, les approches corrélationnelles et qualitatives sont de rigueur. Cependant, au plan des processus psychologiques impliqués dans le phénomène, l'adoption de l'approche expérimentale auprès de populations non à risque de suicide pourrait jeter un éclairage certain sur la question, en permettant d'isoler des causes possibles du phénomène pour en mesurer les effets. À cette fin, des études basées sur l'approche expérimentale pourraient sonder l'influence de certaines caractéristiques des récits de cas de suicides et du type de média sur des processus cognitifs, telles l'interprétation et la mémoire, qui sont des processus impliqués de manière proximale dans le comportement humain. Dans cette lignée, des études appuyées sur cette même approche pourraient examiner le jeu d'interaction potentiel entre les caractéristiques des stimuli médiatiques et différents traits de personnalité des récepteurs.

Dans les trente dernières années, la société a connu une autre révolution technologique importante, soit la venue des ordinateurs et de l'Internet. Cette révolution a donné lieu à une diffusion plus rapide et permet un accès plus facile à de l'information sur le suicide (Tam et al., 2007), mais elle a aussi ouvert la possibilité d'être témoin, en temps réel, du suicide de personnes ou de la déclaration de leur intention de mettre fin à leurs jours. Des études récentes nous enseignent que les sites de socialisation virtuels sont des 
plates-formes de communication propices à l'observation de telles conduites suicidaires (Horne et Wiggins, 2009; Ozawa-de Silva, 2008). De ce fait, il est logique que l'Internet, comme nouveau média de masse, soit appelé, si cela ne l'est déjà, à être associé à des suicides en grappes. L'événement qui a touché la petite municipalité de Bridgend, en Grande-Bretagne, où sept jeunes, dont plusieurs fréquentaient le même site de socialisation virtuelle, se sont enlevés la vie sur une période de 12 mois, en 20072008, est peut-être un nouveau pendant du phénomène de «l'effet Werther ». Ainsi, devant l'évolution rapide des technologies de communication de masse, il est urgent d'intensifier la recherche sur le lien entre les médias de masse et le suicide.

\section{Bibliographie}

ASHTON, J.R. et S.P. DONNAN (1979). "Suicide by burning - A current epidemic », British Medical Journal, septembre, p. 769770 .

BANDURA, A. (1977). Social Learning Theory, Englewood Cliffs, New Jersey, PrenticeHall.

BERMAN, A.L. (1988). «Fictionnal depiction of suicide in television films and imitation effects ", American Journal of Psychiatry, vol. 145 , n ${ }^{\circ} 8$, p. $982-986$.

BOLLEN, K.A. et D.P. PHILLIPS (1982). "Imitative suicides: A national study of the effects of television news stories », American Sociological Reviews, vol. 47, décembre, p. 802-809.

CHENG, T.A., K. HAWTON, T.H.H. CHEN, A.M.F. YEN et al. (2007). "The influence of media coverage of a celebrity suicide on subsequent suicide attempts ", Journal of Clinical Psychiatry, vol. 68, no 6, p. 862-866.

CHIU, S.-H., H.C. KO et J.Y.W. WU (2007). «Depression moderated the effect of exposure to suicide news on suicidality among college students in Taiwan ", Suicide and Life-Threatening Behavior, vol. 37, no 5, p. 585-592.

DORON, A., D. STEIN, Y. LEVINE et al. (1998). «Physiological reactions to a suicide film: Suicide attempters, suicide ideators, and non suicidal patients ", Suicide and LifeThreatening Behavior, vol. 28, no 3, p. 309314

ETZERSDORFER, E., M. VORACEK et G. SONNECK (2004). "A dose-response relationship between imitational suicides and newspaper distribution ", Archives of Suicide Research, vol. 8, p. 137-145.

FEKEE, S. et E. MACSAI (1990). "Hungarian suicidal models : Past and present», dans G. FERRARI, M. BELLINI et P. CREPET (dir.), Suicidal Behavior and Risk Factors, Bologne, Italie, Monduzzi Editore, p. 149156.

FEKETE, S. et A. SCHMIDTKE (1996). «Suicidal models - Their frequency and role in suicide attempters, non suicidal psychiatric patients and normal control case: A com- parative German-Hungarian study », Omega, vol. 33, p. 233-241.

FREI, A., T. SCHENKER, A. FINZEN et al. (2003). "The Werther effect and assisted suicide », Suicide and Life-Threatening Behavior, vol. 33, n 2, p. 192-200.

GOETHE, J.W. (1968 [1774]). Les souffrances du jeune Werther, présentation et traduction par J.-F. ANGELLOZ, Paris, Flammarion.

GIBBONS, R.D., D.C. CLARK et J. FAWCETT (1990). "A statistical method for evaluating suicide clusters and implementing cluster surveillance ", American Journal of Epidemiology, vol. 132, suppl. 1, p. 183-191.

GILES, D. (2003). Media Psychology, Mahwah, New Jersey, Erlbaum.

GOULD, M.S. (2001). "Suicide and the media", Annals of the New York Academy of Sciences, vol. 932, n 1 , p. 200-224.

GOULD, M.S. et D. SHAFFER (1986). «The impact of suicide in television movies ", The New England Journal of Medicine, vol. 315, $\mathrm{n}^{\mathrm{o}} 11, \mathrm{p} \cdot$ 690-694.

GOULD, M.S., D. SHAFFER et M. KLEINMAN (1988). "The impact of suicide in television movies: Replication and commentary », Suicide and Life-Threatening Behavior, vol. $18, \mathrm{n}^{\circ} 1$, p. 90-99.

GOULD, M.S., S. WALLEINSTEIN et L. DAVIDSON (1989). "Suicide clusters: A critical review », Suicide and Life-Threatening Behavior, vol. 19, $\mathrm{n}^{\circ}$ 1, p. 17-29.

GOULD, M.S., S. WALLEINSTEIN, M. KLEINMAN et al. (1990). «Suicide clusters: An examination of age-specific effects », American Journal of Public Health, vol. 80, p. 211-212.

HARRIS, R.J. (1989). A Cognitive Psychology of Mass Communication, Hillsdale, New Jersey, Erlbaum.

HASSAN, R. (1995). «Effects of newspaper stories in the incidence of suicide in Australia: A research note», Australian and New Zealand Journal of Psychiatry, vol. 29, p. 480-483.

HAWTON, K. et K. WILLIAMS (2001). "The connection between media and suicidal behaviour warrants serious attention », Crisis, vol. 22, no 4, p. 137-140.

HAZELL, P. (1993). «Adolescent suicide clusters: Evidence, mechanisms and prevention », Australian and New Zealand Journal of Psychiatry, vol. 27, no 4, p. 653-665.

HITTNER, J.B. (2005). "How robust is the Werther effect? A re-examination of the suggestion-imitation model of suicide», Mortality, vol. 10, $\mathrm{n}^{\circ}$ 3, p. 193-200.

HORNE, J. et S. WIGGINS (2009). «Doing being on the edge: Managing the dilemma of being authentically suicidal in an online forum», Sociology of Health \& Illness, vol. $31, \mathrm{n}^{\circ} 2$, p. 1-15.

JONAS, K. (1992). «Modeling and suicide: A test of the Werther effect », British Journal of Social Psychology, vol. 31, p. 295-306.

JOINER, T.R. Jr. (1999). «The clustering and contagion of suicide ", Current Directions in Psychological Science, vol. 8, no 3, p. 89-92.
KESSLER, R.C., G. DOWNEY,J.R. MILAVSKY et H. STIPP (1988). "Clustering of teenage suicides after television news stories about suicides : A reconsideration ", American Journal of Psychiatry, vol. 145, $\mathrm{n}^{\circ} 11$, p. 13791383.

LITTMANN, S.K. (1985). « Suicide epidemics and newspaper reporting », Suicide and LifeThreatening Behavior, vol. 15, n 1, p. 43-50.

MARCHAND, P. (2004). Psychologie Sociale des Médias, Cedex, France, Presses universitaires de Rennes.

MARTIN, G. (1996). «The influence of television suicide in a normal adolescent population ", Archives of Suicide Research, vol. 2, p. 103-117.

MARTIN, G. et L. KOO (1997). "Celebrity suicide: Did the death of Kurt Cobain influence young suicides in Australia? ", Archives of Suicide Research, vol. 3, p. 187-198.

MISHARA, B.L. et M. TOUSIGNANT (2004). Comprendre le suicide, Québec, Presse de l'Université du Québec.

O'CARROLL, P.W., J.A. MERCY et J.A. STEWARD (1988). "CDC recommendations for a community plan for the prevention and containment of suicide clusters ", Morbidity and Mortality Weekly Report, vol. 37, suppl. 6, p. 1-12.

OZAWA-DE SILVA, C. (2008). «Too lonely to die alone: Internet suicide pacts and existential suffering in Japan ", Culture, Medicine, and Psychiatry, vol. 32, $\mathrm{n}^{\circ}$ 4, p. 516-551.

PHILLIPS, D.P. (1974). "The influence of suggestion on suicide: Substantive and theoretical implications of the Werther effect», American Sociological Review, vol. 39, p. 340-354.

PHILLIPS, D.P. et M.S. CARSTENSEN (1986). «Clustering of teenage suicides after television news stories about suicide», The New England Journal of Medicine, vol. 315, p. 685-689.

PHILLIPS, D.P., K. LESYNA et D.J. PAIGHT (1992). "Suicide and the media», dans R.W. MARIS et al. (dir.), Assessement and Prediction of Suicide, New York, Guilford Press, p. 499-519.

PHILLIPS, D.P. et D.J. PAIGHT (1987). «The impact of televised movies about suicide: A replicative study ", The New England Journal of Medicine, vol. 317, n 13 , p. 809-811.

PIRKIS, J. et R.W. BLOOD (2001a). « Suicide and the media. Part I: Reportage in nonfictional media», Crisis, vol. 22, n ${ }^{\circ} 4$, p. 146154.

PIRKIS, J. et R.W. BLOOD, (2001b). « Suicide and the media. Part II : Portrayal in fictional media », Crisis, vol. 22, no 4, p. 155-162.

PIRKIS, J., P.M. BURGESS, C. FRANCIS et al. (2006). "The relationship between media reporting of suicide and actual suicide in Australia», Social Science \& Medicine, vol. 62 , p. 2874-2886.

PLATT, S. (1987). «The aftermath of Angie's overdose: Is soap (opera) damaging to your health ?», British Medical Journal, vol. 294, p. 954-957. 
POST, R.M. (1992). «Transduction of psychosocial stress into the neurobiology of recurrent affective disorder », American Journal of Psychiatry, vol. 149, p. 999-1009.

POST, R.M. et S.R.B. WEISS (1995). «The neurobiology of treatment-resistant mood disorders ", dans F.E. BLOOM et F.J. KUPFER (dir.), Psychopharmacology: The Fourth Generation of Progress, New York, Raven Press, p. $1155-1170$.

POULIOT, L. (2001). L'influence de la structure et de la valence émotionnelle du récit filmique sur la reconnaissance de l'information, thèse de doctorat, Montréal, Université du Québec à Montréal.

SEVERIN, W.J. et J.W. TANKARD Jr. (1988). Communication Theories: Origins, Methods, Uses, $2^{\mathrm{e}}$ éd., New York, Longman.

SCHMIDTKE, A. et H. HÄFNER (1988).

"The Werther effect after television films: New evidence for an old hypothesis », Psychological Medicine, vol. 18, p. 665-676.

SCHMIDTKE, A. et SCHALLER, S. (1998). "What do we know about media effects on imitation of suicidal behaviour: State of the art», dans D. DE LEO et R.F.W. DIEKSTRA (dir.), Suicide Prevention: A Holistic Approach, Pays-Bas, Kluwer Academic Publishers, p. 121137.
SHOVAL, G., G. ZALSMAN, J. POLAKEVITCH et al. (2005). "Effect of the broadcast of a television documentary about a teenager's suicide in Israel on suicidal behavior and methods », Crisis, vol. 26, nº 1, p. 20-24.

SINGER, D.G., et J.L. SINGER (2001). Handbook of Children and the Media, Thousand Oaks (CA), Sage.

STACK, S. (1987). "Celebrities and suicide: A taxonomy and analysis ", American Sociological Review, vol. 52, p. 402-411.

STACK, S. (1996). «The effect of the media on suicide: Evidence from Japan, 1955-1985», Suicide and Life-Threatening Behavior, vol. $26, \mathrm{n}^{\circ} 2$, p. 132-142.

STACK, S. (2002). " Media coverage as a risk factor in suicide », Injury Prevention, vol. 8, p. 30-32.

STACK, S. (2003). « Media coverage as a risk factor in suicide ", Journal of Epidemiology and Community Health, vol. 57, n ${ }^{\circ} 4$, p. 238240.

STACK, S. (2005). "Suicide in the media: A quantitative review of studies based on nonfictional stories", Suicide and LifeThreatening Behavior, vol. 35 , n ${ }^{\circ} 2$, p. 121133.
TAM, J., W.S. TANG et D.J.S. FERNANDO (2007). "The Internet and suicide: A doubleedged tool », European Journal of Internal Medicine, vol. 18, p. 453-455.

TOUSIGNANT, M., B.L. MISHARA, A. CAILLAUD et al. (2005). "The impact of media coverage of the suicide of a well-known Quebec reporter: The case of Gaétan Girouard», Social Science \& Medicine, vol. 60, p. 1919-1926.

YIP, P.S., K.W. FU, K.C. YANG et al. (2006). "The effects of a celebrity suicide on suicide rates in Hong Kong», Journal of Affective Disorders, vol. 93, p. 245-252.

ZAHL, D.L. et K. HAWTON, (2004). «Media influences on suicidal behaviour: An interview study of young people», Behavioural and Cognitive Psychotherapy, vol. 32, p. 189198.

\section{Notes}

1. L'auteure tient à remercier Louise Sansoucy, Steve Carrière et Éveline Pilon pour leur contribution à la recension des écrits qui ont servi de toile de fond à cet article.

2. Pour un traitement en profondeur de ces questions, voir: Giles, 2003; Harris, 1989; Marchand, 2004; Pouliot, 2001; Severin et Tankard, 1988. 